\title{
Signals of Significantly Increased Vaccine Breakthrough, Decreased Hospitalization Rates, and Less Severe Disease in Patients with Coronavirus Disease 2019 Caused by the Omicron Variant of Severe Acute Respiratory Syndrome Coronavirus 2 in Houston, Texas
}

Paul A. Christensen, ${ }^{* \dagger \ddagger}$ Randall J. Olsen, ${ }^{* \dagger \ddagger}$ S. Wesley Long, ${ }^{* \dagger \ddagger}$ Richard Snehal, ${ }^{*}$ James J. Davis, ${ }^{\S \llbracket}$ Matthew Ojeda Saavedra, ${ }^{*}$ Kristina Reppond, * Madison N. Shyer, , Jessica Cambric, * Ryan Gadd, * Rashi M. Thakur, ${ }^{*}$ Akanksha Batajoo, * Regan Mangham, ${ }^{*}$ Sindy Pena, ${ }^{*}$ Trina Trinh, ${ }^{*}$ Jacob C. Kinskey, ${ }^{*}$ Guy Williams, ${ }^{*}$ Robert Olson,,${ }^{\S}$ Jimmy Gollihar, ${ }^{\dagger}$ and James M. Musser ${ }^{*}+\ddagger$

From the Laboratory of Molecular and Translational Human Infectious Diseases Research* and Laboratory of Antibody Discovery and Accelerated Protein Therapeutics, ${ }^{\dagger}$ Center for Infectious Diseases, Houston Methodist Research Institute and Department of Pathology and Genomic Medicine, Houston Methodist Hospital, Houston, Texas; the Department of Pathology and Laboratory Medicine, ${ }^{\ddagger}$ Weill Cornell Medical College, New York, New York; the Consortium for Advanced Science and Engineering, ${ }^{\S}$ University of Chicago, Chicago, Illinois; and the Computing, Environment and Life Sciences, ${ }^{\Uparrow}$ Argonne National Laboratory, Lemont, Illinois

Accepted for publication January 20, 2022.

Address correspondence to James M. Musser, M.D., Ph.D., Department of Pathology and Genomic Medicine, Houston Methodist Research Institute, 6565 Fannin St., Ste. B490, Houston, TX 77030. E-mail: jmmusser@houstonmethodist. org.
Genetic variants of severe acute respiratory syndrome coronavirus 2 (SARS-CoV-2) continue to dramatically alter the landscape of the coronavirus disease 2019 (COVID-19) pandemic. The recently described variant of concern designated Omicron (B.1.1.529) has rapidly spread worldwide and is now responsible for the majority of COVID-19 cases in many countries. Because Omicron was recognized recently, many knowledge gaps exist about its epidemiology, clinical severity, and disease course. A genome sequencing study of SARS-CoV-2 in the Houston Methodist health care system identified 4468 symptomatic patients with infections caused by 0micron from late November 2021 through January 5, 2022. Omicron rapidly increased in only 3 weeks to cause $90 \%$ of all new COVID-19 cases, and at the end of the study period caused $98 \%$ of new cases. Compared with patients infected with either Alpha or Delta variants in our health care system, Omicron patients were significantly younger, had significantly increased vaccine breakthrough rates, and were significantly less likely to be hospitalized. Omicron patients required less intense respiratory support and had a shorter length of hospital stay, consistent with on average decreased disease severity. Two patients with Omicron stealth sublineage BA.2 also were identified. The data document the unusually rapid spread and increased occurrence of COVID-19 caused by the Omicron variant in metropolitan Houston, Texas, and address the lack of information about disease character among US patients. (Am J Pathol 2022, 192: 642-652; https://doi.org/ 10.1016/j.ajpath.2022.01.007)
Supported by the Houston Methodist Academic Institute Infectious Diseases Fund; and in part with funds from the National Institute of Allergy and Infectious Diseases, NIH, Department of Health and Human Services, under contract 75N93019C00076 (J.J.D.).
P.A.C., R.J.O., and S.W.L. contributed equally to this work.

Disclosures: None declared. 
Over the past 14 months, the Alpha and Delta variants of concern (VOCs) of severe acute respiratory syndrome coronavirus 2 (SARS-CoV-2) have caused two distinct coronavirus disease 2019 (COVID-19) surges in the United States, Southeast Asia, Europe, and elsewhere (https://www.cdc. gov/coronavirus/2019-ncov/cases-updates/variant-survei llance/variant-info.html, last accessed December 30, 2021; https://www.gov.uk/government/collections/newsars-cov-2-variant, last accessed December 30, 2021), and remodeled the landscape of human behavior and many societies. Delta replaced the Alpha variant as the cause of virtually all COVID-19 in many countries (https://www.who.int/publications/m/item/weekly-epidemio logical-update-on-covid-19-13-july-2021, last accessed August 18, 2021; https://www.ons.gov.uk/peoplepopulati onandcommunity/healthandsocialcare/conditionsanddise ases/bulletins/coronaviruscovid19infectionsurveypilot/ 9july2021, last accessed August 18, 2021).

At the start of the pandemic almost 2 years ago, the Houston Methodist health care system instituted a comprehensive and integrated population genomics project designed to sequence all SARS-CoV-2 samples causing COVID-19 in patients cared for at our facilities, which include eight hospitals located throughout the metroplex. The project was implemented when the initial Houston Methodist COVID-19 case was diagnosed at the end of February 2020, and has continued unabated. ${ }^{1-7}$ This project was facilitated by the existence of a single large diagnostic laboratory that serves the entire system and is seamlessly integrated with a research institute with extensive genomics expertise and capacity. A key goal was to comprehensively map the population genomics, trajectory, and other features of the pandemic in metropolitan Houston, Texas, with a population size of approximately 7.2 million. Houston is the fourth largest city in the United States, is the most ethnically diverse metropolitan area in the country, and is a major port of entry. To date, SARS-CoV-2 genomes have been sequenced from $>70,000$ patient samples. Many features of four distinct SARS-CoV-2 waves in Houston have been described. $^{2-6}$

The successes of rapid SARS-CoV-2 vaccine development and documented efficacy, coupled with the significant downturn of the disease wave caused by Delta in Houston and elsewhere in fall $2021,{ }^{6}$ suggested that the pandemic was abating. However, the identification of a new VOC designated B.1.1.529 and known as Omicron that has spread rapidly in South Africa and the United Kingdom has tempered this optimism. ${ }^{8-10}$ Inasmuch as Omicron was recognized recently, and much is not known about its epidemiology and clinical characteristics and course, we used our integrated infrastructure in an effort to address the lack of information available for US Omicron patients. Genome sequencing identified 4468 COVID-19 patients with symptomatic disease caused by Omicron in the Houston Methodist health care system beginning in late November 2021 and ending January 5, 2022. In 3 weeks, Omicron spread throughout the Houston metropolitan region to become the cause of $90 \%$ of new COVID-19 cases, and at the end of the study period caused $98 \%$ of all new cases. Compared with patients infected with either Alpha or Delta variant and cared for in our system, significantly fewer Omicron patients were hospitalized, and those who were hospitalized required significantly less intense respiratory support and had a shorter length of stay. Our findings are consistent with decreased disease severity among Houston Methodist Omicron patients. Many factors undoubtedly have contributed, including but not limited to increased vaccination uptake, population immunity, and patient demographics, such as younger age. The extent to which our findings translate to other cities and other patient populations, including children, is unknown. These data expand on our initial Omicron work ${ }^{7}$ and address the lack of information about disease character among US patients with COVID-19 caused by this VOC.

\section{Materials and Methods}

\section{Patient Specimens}

Specimens were obtained from patients registered at Houston Methodist facilities (eg, hospitals and urgent care centers) and institutions in the Houston metropolitan region that use our laboratory services. The great majority of individuals had signs or symptoms consistent with COVID-19 disease. For analyses focusing on patients with COVID-19 caused by the Omicron variant, samples obtained from November 27, 2021, through January 5, 2022, were used. This time frame was chosen because it represents the period during which an Omicron variant was first identified in our health care system and the last date of specimen collection used to generate genome sequence data for this article. Note that the genome data were generated for two distinct sampling periods. The first period included November 27, 2021, through December 23, 2021; and the second period included samples obtained between December 30, 2021, and January 5, 2022. This discontinuous sampling strategy was used in an effort to obtain the most up-to-date data available for inclusion in this study. Because of the substantial number of positive specimens obtained daily in the December 24, 2021, to December 29, 2021, period (sometimes exceeding 1500), it was not possible to sequence most of the samples collected during this period for inclusion in the study.

For analyses comparing features of patients infected with the Omicron VOC and Alpha and Delta VOCs, all patients documented to be infected with these variants in the Houston Methodist system were studied. The study included 40,991 unique patients identified in this time frame for whom we had SARS-CoV-2 genome sequences. The work was approved by the Houston Methodist Research Institute Institutional Review Board (IRB1010-0199). 


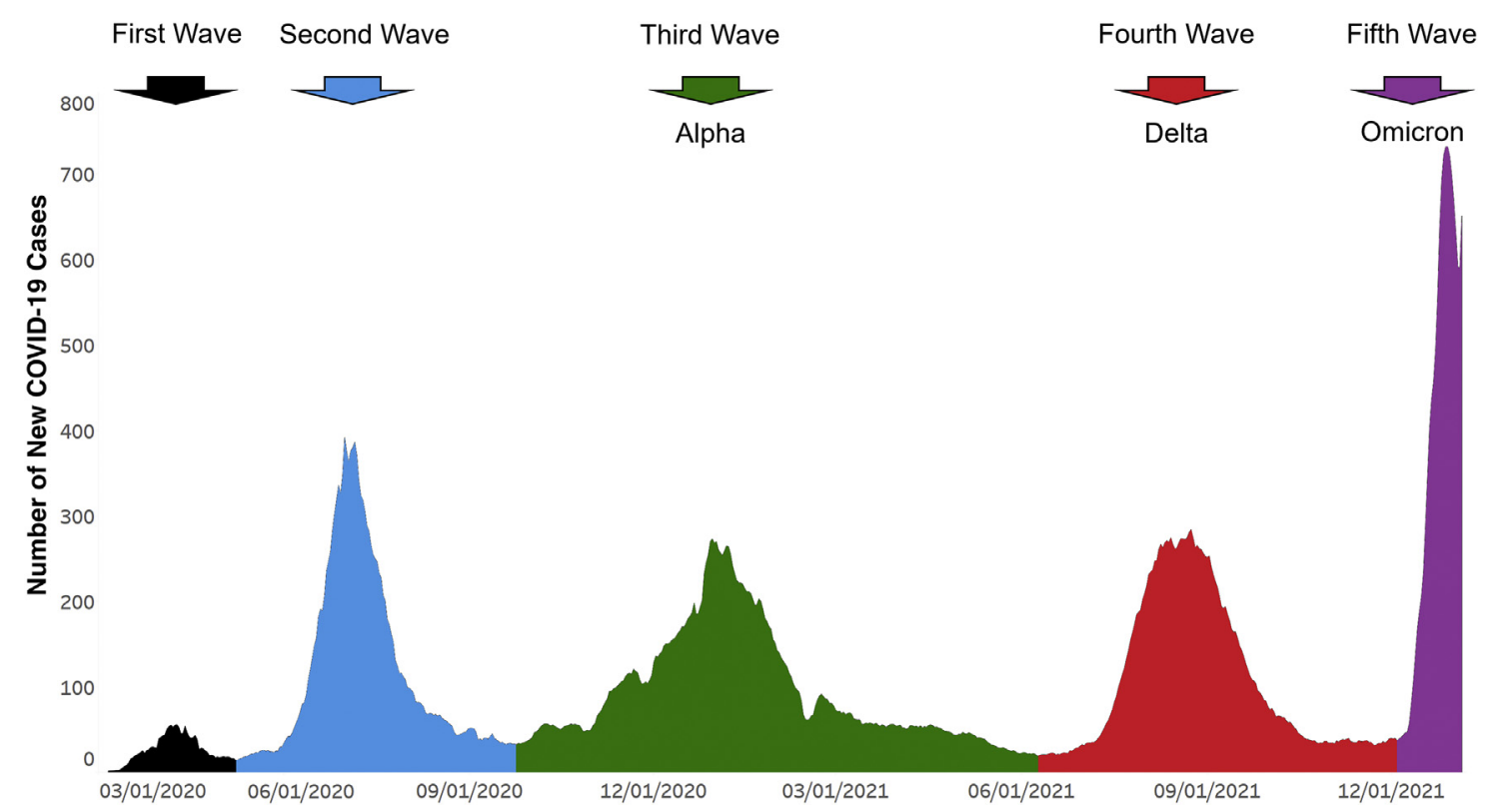

Figure 1 Epidemiologic curve showing five COVID-19 disease waves in Houston Methodist patients. Number of new COVID-19 cases ( $y$ axis) totals are shown as a \pm 3 -day moving average. Each of the five waves is shown in a different color. The first and second waves were composed of a heterogeneous array of SARS-CoV-2 genotypes. The Alpha variant of concern (VOC) shown in the third wave, the Delta VOC shown in the fourth wave, and the Omicron VOC shown in the fifth wave indicate their numeric prominence in those waves. The figure should not be interpreted to mean that all cases in the third, fourth, and fifth waves were caused by Alpha, Delta, and Omicron VOCs, respectively. Rather, they are the dominant single VOCs causing disease in Houston Methodist system patients in those waves. The fifth wave shown includes data through January 5, 2022. The figure was generated with Tableau version 2021.2 .7 (Tableau Software, LLC, Seattle, WA), and is adapted with permission from the version presented in Christensen et al. ${ }^{6}$ The curve is essentially superimposable on COVID19 activity in all metropolitan Houston, Texas.

\section{SARS-CoV-2 Molecular Diagnostic Testing}

Specimens obtained from symptomatic patients with a suspicion for COVID-19 disease were tested in the Molecular Diagnostics Laboratory at Houston Methodist Hospital using assays granted Emergency Use Authorization from the US Food and Drug Administration (https://www.fda.gov/medical-devices/ emergency-situations-medical-devices/faqs-diagnostic-testingsars-cov-2\#offeringtests, last accessed June 7, 2021). Multiple molecular testing platforms were used, including the COVID19 test or RP2.1 test with BioFire Film Array instruments (BioFire Diagnostics, Salt Lake City, UT), the Xpert Xpress SARS-CoV-2 test using Cepheid GeneXpert Infinity or Cepheid GeneXpert Xpress IV instruments (Cepheid, Sunnyvale, CA), the Cobas SARS-CoV-2 and Influenza A/B Assay using the Roche Liat system (Roche Diagnostics, Indianapolis, IN), the SARS-CoV-2 Assay using the Hologic Panther instrument (Hologic, Marlborough, MA), the Aptima SARS-CoV-2 Assay using the Hologic Panther Fusion system (Hologic), the Cobas SARS-CoV-2 test using the Roche 6800 system, and the SARSCoV-2 assay using Abbott Alinity $m$ instruments (Abbott Molecular, Des Plaines, IL). Virtually all tests were performed on material obtained from nasopharyngeal swabs immersed in universal transport media; oropharyngeal or nasal swabs, bronchoalveolar lavage fluid, or sputum treated with dithiothreitol were sometimes used. Standardized specimen collection methods were used (https://vimeo.com/396996468/ 2228335d56, last accessed June 7, 2021).

\section{SARS-CoV-2 Genome Sequencing, Genome Analysis, and Identification of Variants}

We sequenced the SARS-CoV-2 genome of $>90 \%$ of all positive cases in the Houston Methodist health care system during the two sampling periods studied. Libraries for whole SARS-CoV-2 genome sequencing were prepared according to version 4 (https://community.artic.network/t/sars-cov-2version-4-scheme-release/312, last accessed August 19, 2021) of the ARTIC nCoV-2019 sequencing protocol. The semi-automated workflow used has been described previously. ${ }^{2-6}$ Sequence reads were generated with an Illumina (San Diego, CA) NovaSeq 6000 instrument.

Viral genomes were assembled with the BV-BRC SARSCov2 assembly service (https://www.bv-brc.org/app/ ComprehensiveSARS2Analysis, last accessed June 7, 2021, requires registration). The pipeline currently uses seqtk version 1.3-r117 for sequence trimming (https://github. com/lh3/seqtk.git, last accessed December 30, 2021) and minimap version 2.17 for aligning reads against the Wuhan-Hu-1 (NC_045512.2) reference genome. Samtools version 1.11 was used for sequence and file manipulation, where maximum depth and minimum depth parameters in mpileup were set to 8000 and 3, respectively. iVar version 1.3.1 was used for primer trimming and variant calling. Genetic lineages, VOCs, and variants of interest were identified on the basis of genome sequence data and designated by Pangolin version 3.1.17 with pangoLEARN 
module 2021-12-06 (https://cov-lineages.org/resources/ pangolin.html, last accessed December 12, 2021). Genome data used in this study have been deposited to Global Initiative on Sharing Avian Influenza Data (GISAID; www.gisaid.org) (Supplemental Table S1).

\section{S-Gene Target-Failure Assay}

An $S$-gene target-failure (SGTF) assay (TaqPath COVID-19 Combo Kit; Thermo Fisher, Inc., Waltham, MA) was used as a surrogate marker for the Omicron VOC for some specimens collected between December 18, 2021, and January 5, 2022. From November 1, 2021, onward, only Delta and Omicron were documented to be circulating in metropolitan Houston, based on whole-genome sequence data. Patient samples were first tested in the clinical Molecular Diagnostics Laboratory using a RT-PCR assay with an Emergency Use Authorization, as described above. The SARS-CoV-2-positive samples were then tested with the SGTF assay, according to the manufacturer's instructions to infer an Omicron or not-Omicron lineage. That is, the SGTF assay was only performed on samples known to be positive for SARS-CoV-2. Samples yielding amplification of the $S$ gene were classified as a Delta variant. The SGTF data were validated on the basis of comparing the results with our extensive genome sequence data.

\section{Patient Metadata and Geospatial Analysis}

Patient metadata were acquired from the electronic medical record by standard informatics methods. Figures showing geospatial distribution of spread for Omicron were generated with Tableau version 2021.2.7 (Tableau Software, LLC, Seattle, WA) using patient home address zip codes. A vaccination breakthrough case was defined as a PCRpositive sample from a patient obtained $>14$ days after full vaccination (eg, both doses of the Pfizer or Moderna mRNA vaccines) was completed. A booster vaccination breakthrough case was defined as a PCR-positive sample from a patient obtained $>14$ days after receiving a third vaccine dose. For some cases, manual chart review was conducted to resolve discrepancies or clarify ambiguities.

\section{Results}

\section{Omicron Epidemiologic Wave}

The first Houston Methodist patient infected with the Omicron variant was identified at the end of November 2021, a time when the Delta VOC was responsible for all COVID19 cases in metropolitan Houston. ${ }^{6}$ During this period, the metropolitan area was experiencing a steady decrease in total number of new COVID-19 cases (Figures 1 and 2).

Omicron increased in frequency unusually rapidly over a 3-week period in December (Figures 1 and 2). By December 23 , the genome sequence data showed that Omicron accounted for $>90 \%$ of all new COVID-19 cases in our health care system (Figure 2). The estimated case doubling time during this 3-week period was approximately 1.8 days (Figure 2), which means that Omicron increased in relative frequency approximately three times faster than Delta had increased in our area, ${ }^{6}$ an unprecedented trajectory for SARSCoV-2 infections. By January 5, 2022, the Omicron variant caused $98 \%$ of all new COVID-19 cases diagnosed in our health care system (Figure 2). This represents the fifth wave of COVID-19 cases in metropolitan Houston (Figure 1).

Consistent with extensive infections caused by Omicron in southern Africa and elsewhere (https://www.cdc.gov/ coronavirus/2019-ncov/variants/variant-classifications. html, last accessed December 28, 2021; https://www.gov.uk/ government/collections/new-sars-cov-2-variant, last accessed December 28, 2021), several patients had recent travel histories to countries with a high prevalence of this VOC, suggesting acquisition of virus by some cases from abroad and importation into Houston. However, the vast majority of Omicron patients had no documented travel outside the United States and undoubtedly acquired the infection domestically, either in Houston or elsewhere.

To understand the geospatial distribution of Omicron in metropolitan Houston, patient metadata were acquired from the electronic medical record by standard informatics methods, and home address zip codes were used to visualize virus spread (Figure 2). The 4468 Houston Methodist patients infected with Omicron during this period were distributed widely throughout metropolitan Houston, with 259 different zip codes represented (Figure 2). The widespread distribution of Omicron in the Houston metroplex in an extremely short period of time reflects the ability of this variant to spread unusually rapidly and effectively between individuals and cause symptomatic disease.

\section{Comparison of Omicron, Alpha, and Delta COVID-19 Cases}

There is a considerable lack of detailed information about patients with COVID-19 caused by the Omicron VOC, and data are especially lacking for US patients. Available metadata for all Houston Methodist patients infected with Omicron, Alpha, and Delta VOCs (Tables 1 and 2) were compared. The populations differed significantly in many characteristics, including median age, hospital admission rates, maximum respiratory support, rate of vaccine breakthrough, and median length of stay (Tables 1 and 2).

Patients infected with Omicron were significantly younger than patients infected with Alpha and Delta (Tables 1 and 2). More importantly, Omicron patients were hospitalized significantly less frequently than patients infected with either the Alpha or Delta variant, and had a significantly shorter median hospital length of stay (Tables 1 and 2).

Omicron vaccine breakthrough cases (Tables 1 and 2) were analyzed next. Of the 4468 total Omicron patients for 

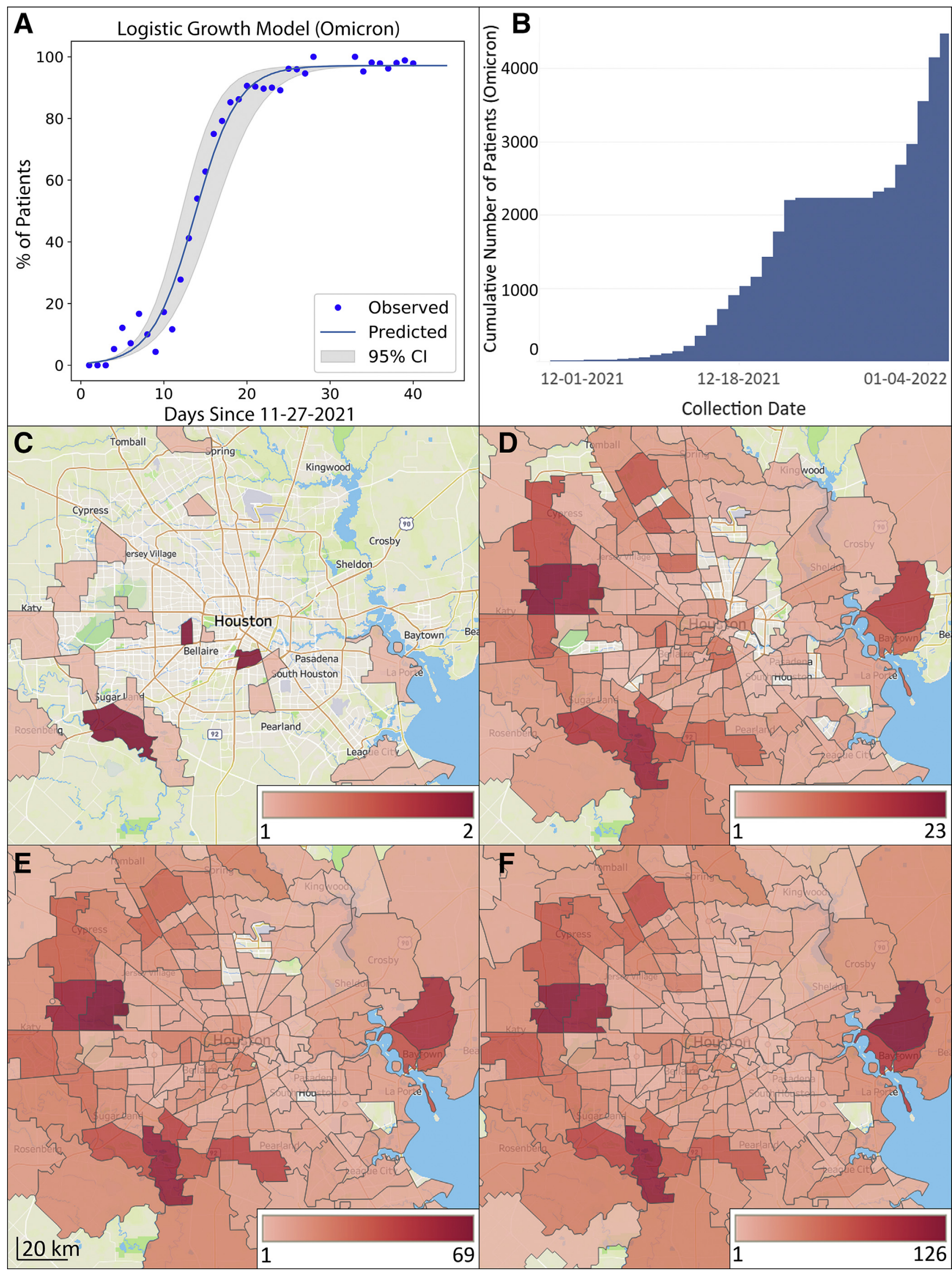
whom whole genome sequence data were available, 2497 (55.9\%) met the CDC definition of vaccine breakthrough cases (Tables 1 and 2). There was no simple relationship between the time elapsed since administration of the second vaccination dose and the date of vaccination breakthrough. These 2497 patients received two doses of either the PfizerBioNTech BNT162b2 $(n=1828 ; 73 \%)$ or the Moderna mRNA-1273 $(n=553 ; 22 \%)$ or one dose of $\mathrm{J} \& \mathrm{~J} / \mathrm{J} a n s s e n$ JNJ-78436735 $(n=115 ; 5 \%)$ vaccine. Vaccine type was not specified for one individual. This distribution reflects the majority use of BNT162b2 vaccination doses in our health system. Compared with patients with either Alpha or Delta VOC, a significantly greater percentage of patients with breakthrough cases had the Omicron VOC $(55.9 \%$ compared with $3.2 \%$ and $24.3 \%$ for Alpha and Delta VOCs, respectively) (Tables 1 and 2). Individuals with breakthrough cases after receiving a third (booster) dose of either the Pfizer-BioNTech BNT162b2 or the Moderna mRNA1273 vaccine were analyzed next. Of the 4468 patients with the Omicron VOC, $711(15.9 \%)$ met this criterion. Consistent with Omicron causing a significantly increased number of vaccine breakthrough cases, many studies have reported that this variant has reduced sensitivity to antibody neutralization in vitro, likely in large part due to the extensive number of amino acid and other structural changes occurring in Omicron spike protein. ${ }^{11-34}$

\section{Spike-Gene Target-Failure Assay}

To estimate Omicron variant frequency in patient samples not yet sequenced, the TaqPath COVID-19 Combo Kit assay (Thermo Fisher) was performed on 1216 samples collected from symptomatic patients between December 18, 2021, and January 5, 2022. In total, 1093 (90\%) of patient samples yielded an RT-PCR result with $S$-gene target-failure indicative of the Omicron variant. These data are consistent with the increasing frequency of new cases of COVID-19 caused by Omicron in our population (Figure 2).

\section{Discovery of Omicron Stealth Sublineage BA.2 in Houston}

The Omicron sublineage BA.2 was first identified in November 2021 in Australia in a patient who had traveled to South Africa (https://github.com/cov-lineages/pangodesignation/issues/359, last accessed December 30, 2021). This sublineage does not have the full set of polymorphisms characteristic of BA.1 (B.1.1.529) and has additional mutations unique to it (https://github.com/cov- lineages/pango-designation/issues/361, last accessed December 30, 2021). One important difference is that sublineage BA.2 lacks the spike gene deletion in the region encoding amino acid 69/70, which means that it will not be detected by the SGTF assay. As a consequence, it is sometimes referred to as the Omicron stealth variant. All full genome sequences present in our large database were inspected, including specimens obtained from symptomatic patients and asymptomatic individuals, and only two members of the BA.2 sublineage were discovered in Houston COVID-19 patients.

\section{Discussion}

This work was conducted to address the relative lack of information about disease character among US patients with COVID-19 caused by the Omicron VOC, and to compare the findings with data available for patients in the Houston Methodist system who had disease caused by the Alpha and Delta VOCs. Information relevant to the massive Omicron wave in metropolitan Houston is described here. In 3 weeks (December 1, 2021, through December 23, 2021), Omicron was first identified in our population and rapidly increased to cause $90 \%$ of all new COVID-19 cases, with an unusually fast case doubling time of 1.8 days. Analysis of samples obtained from December 30, 2021, to January 5, 2022, found that at the end of the sampling period, Omicron caused 98\% of all new COVID-19 cases in our health care system.

The study was based on genome sequence analysis of 4468 Omicron samples taken from socioeconomically, geographically, and ethnically diverse symptomatic patients. Several key findings were made, including the following: i) the Omicron VOC rapidly increased as a cause of COVID-19 and spread throughout the metroplex in an unusually short period of time, far faster than any other SARS-CoV-2 variant; ii) Omicron caused significantly more vaccine breakthrough cases than the Alpha or Delta VOC; iii) Omicron patients were significantly younger than Alpha or Delta patients; iv) significantly fewer Omicron patients required hospitalization compared with Alpha and Delta patients; and v) the median length of stay for hospitalized Omicron patients was significantly shorter than for Alpha and Delta patients, and consistent with this observation, on average the maximum respiratory support required for Omicron patients was significantly less than for Alpha or Delta patients. These findings are largely consistent with many aspects of Omicron data reported from the United Kingdom, South Africa, and Canada, ${ }^{8-10,35-38}$ and are consistent with experimental

Figure 2 Increase in Omicron frequency over time and distribution in metropolitan Houston, Texas. The study time frame was November 27, 2021, through January 5, 2022. A: Omicron logistic growth model. The estimated case doubling time is 1.8 days. B: Cumulative increase in 0micron during the study period; $y$ axis is the cumulative number of new COVID-19 0micron cases. At the end of the study period, Omicron caused $98 \%$ of all COVID-19 cases. The plateau between December 24, 2021, and December 30, 2021, exists because samples obtained during this period were not sequenced due to the massive number of daily positive specimens, as described in Materials and Methods. C-F: Geospatial distribution of Omicron based on home address zip code for each patient. C: November 27 to December 6. D: November 27 to December 16. E: November 27 to December 26. F: November 27 to January 5. Note differences in heat map scale for each panel. Figures were generated using Tableau version 2021.2.7. (Tableau Software, LLC, Seattle, WA). 
Table 1 Summary of Pertinent Patient Metadata for 7617 Unique Patients Infected with Omicron or Alpha Variant

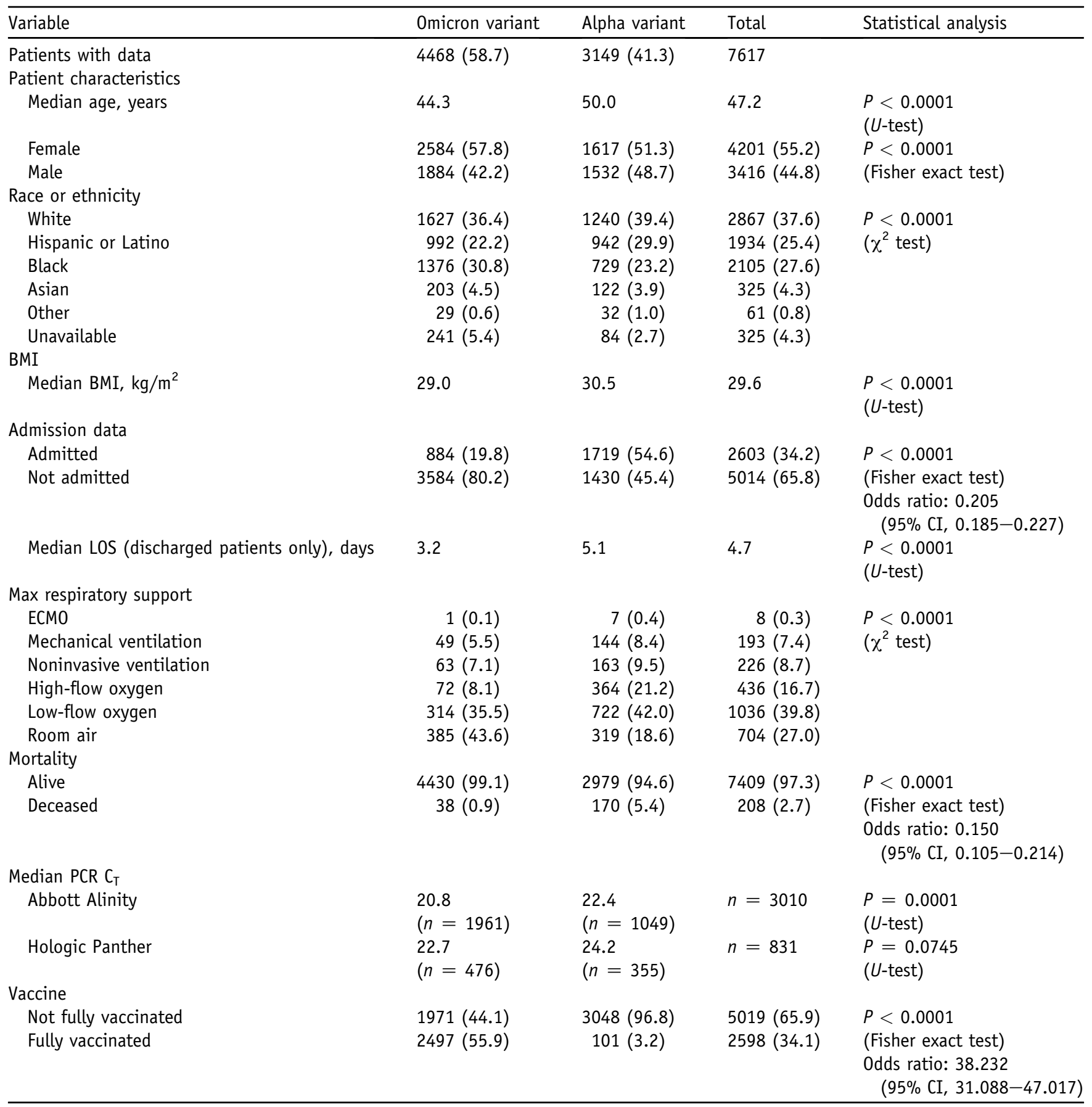

Data are given as number (percentage) of patients, unless otherwise indicated.

BMI, body mass index; ECMO, extracorporeal membrane oxygenation; LOS, length of stay; Max, maximum.

animal infection data suggesting that Omicron causes less severe disease in mice and hamsters. ${ }^{39-43}$ This study was facilitated by a comprehensive and integrated population genomics and epidemiology project $^{2-6}$ implemented at the end of February 2020, when the initial COVID-19 case was diagnosed in the Houston Methodist health care system.

Several questions arise from these findings (namely, the underlying causes for the differences observed in Omicron compared with Alpha and Delta patients). Increased vaccine breakthrough cases may occur due to serologic and structural differences in Omicron relative to Alpha and Delta. It is also possible that waning of immunity is a contributing factor as well. Currently, no serologic or other data are available that could address this possibility in our patients. As noted above, ample in vitro and animal infection model data have accumulated, suggesting that Omicron is less virulent than Delta or Alpha VOC. We speculate that the lower age of Omicron patients may be attributable to a 
Table 2 Summary of Pertinent Patient Metadata for 20,196 Unique Patients Infected with Omicron or Delta Variant

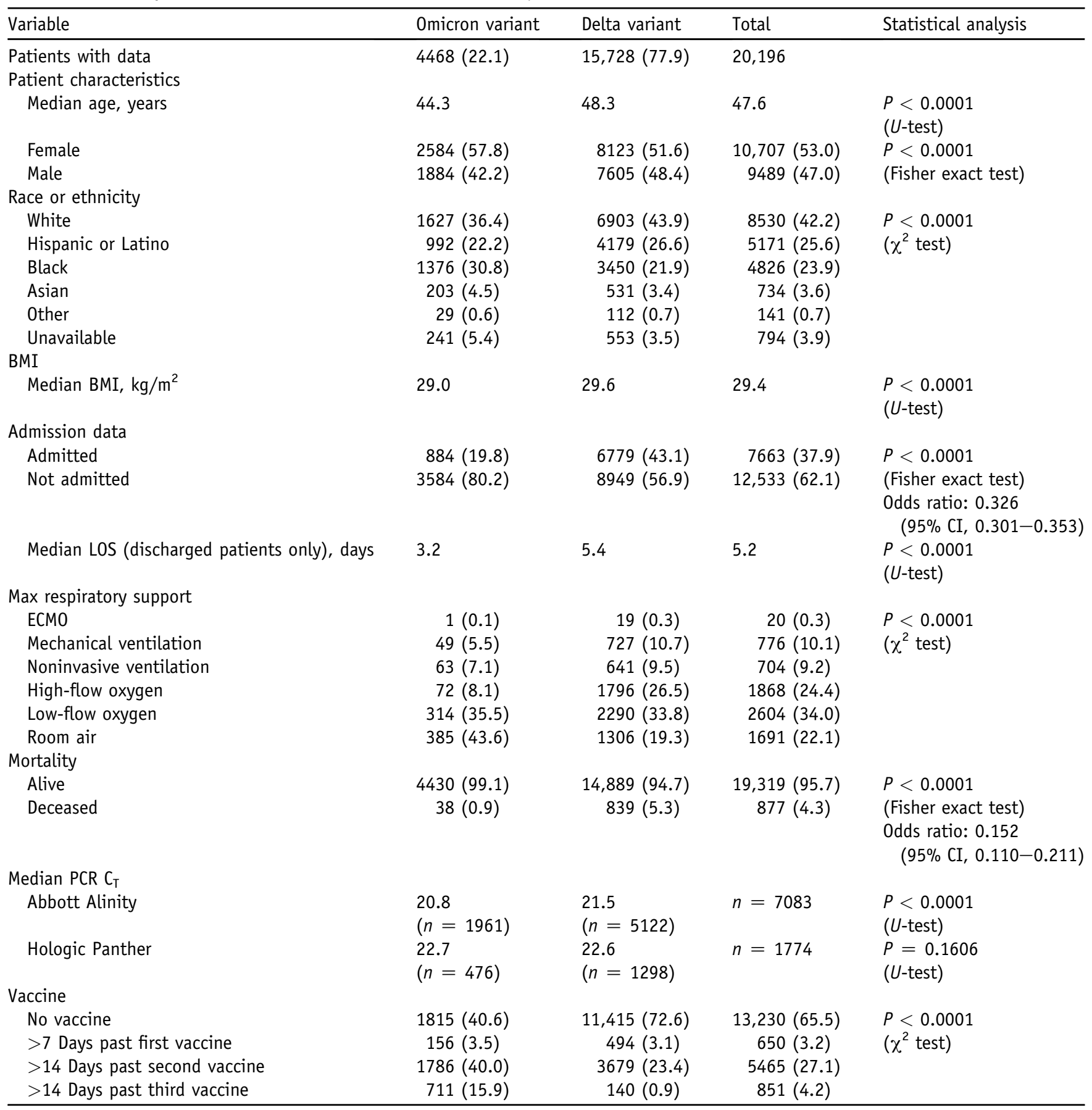

Data are given as number (percentage) of patients, unless otherwise indicated.

BMI, body mass index; ECMO, extracorporeal membrane oxygenation; LOS, length of stay; Max, maximum.

disproportionately greater likelihood of risky behaviors in the younger population (eg, less mask wearing and less social distancing). Regardless, additional studies are required to gain more information about factors contributing to the differences between Alpha, Delta, and Omicron patients that were identified in this study.

Because the genome of approximately $90 \%$ of SARSCoV-2 causing COVID-19 in our diverse Houston
Methodist patient population is sequenced, and has been done for almost 2 years, the composition of this virus is continuously being monitored in a major US metroplex. This affords the opportunity to rapidly assess changes in SARS-CoV-2 population genomic structure in the fourth largest city in the United States. However, this study has several limitations. Although genomes of SARS-CoV-2 causing $90 \%$ of all Houston Methodist COVID-19 cases 
in the study period were sequenced, this sample represents only approximately $5 \%$ of cases reported in the metropolitan region. This patient population underrepresents some demographic groups (eg, homeless individuals and pediatric patients). The samples sequenced in this study were obtained from symptomatic individuals, which means that it is possible that Omicron subvariants or features preferentially represented in asymptomatic individuals were not identified. It is likely that this study included some patients where Omicron was detected on hospital admission but was incidental to the primary cause of admission.

The identification of two asymptomatic individuals with the Omicron stealth sublineage BA.2 is potentially concerning and stresses the importance of using whole-genome sequencing to study patient samples. This sublineage lacks the spike gene deletion corresponding to amino acids 69 and 70 and is not detected by some commonly used assays. Sublineage BA.2 now accounts for approximately 5\% of COVID-19 in the United Kingdom, which means that it has the ability to successfully transmit and cause disease. ${ }^{44}$ It will be important to determine if this SARS-CoV-2 genotype increases in frequency in metropolitan Houston as additional genome sequencing is conducted on samples from our patient population.

In the aggregate, these data add critical new information to features of Omicron genomic epidemiology and patient characteristics in the United States. Furthermore, the present study highlights the importance of analyzing SARS-CoV-2 genome data integrated with patient metadata and stresses the need to continue to do this in near real time as the Omicron surge continues, the virus evolves, and new variants with potentially altered fitness and biomedically relevant phenotypes are generated. Analyses of this type are also important in the context of vaccine formulation and long COVID-19, an increasing health and economic problem globally. Finally, the strategy we have used in this and previous studies $^{2-6}$ is readily applicable to future infectious disease problems that warrant special attention.

\section{Acknowledgments}

We thank Drs. Marc Boom and Dirk Sostman for ongoing support; and Dr. Sasha M. Pejerrey for editorial contributions.

\section{Author Contributions}

P.A.C., R.J.O., S.W.L., and J.M.M. had full access to all study data and take responsibility for the integrity of the data and the accuracy of data analysis; J.M.M., P.A.C., R.J.O., and S.W.L. conceived and designed the study; all authors acquired, analyzed, or interpreted data and wrote the manuscript; P.A.C. analyzed data; J.M.M. and J.J.D. obtained funding; J.M.M. provided overall supervision;
P.A.C., R.J.O., and S.W.L. contributed equally and are co-first authors.

\section{Supplemental Data}

Supplemental material for this article can be found at http://doi.org/10.1016/j.ajpath.2022.01.007.

\section{References}

1. Dhar MS, Marwal R, Vs R, Ponnusamy K, Jolly B, Bhoyar RC, et al: Genomic characterization and epidemiology of an emerging SARSCoV-2 variant in Delhi, India. Science 2021, 374:995-999

2. Long SW, Olsen RJ, Christensen PA, Bernard DW, Davis JJ, Shukla M, Nguyen M, Saavedra MO, Yerramilli P, Pruitt L, Subedi S, Kuo HC, Hendrickson H, Eskandari G, Nguyen HAT, Long JH, Kumaraswami M, Goike J, Boutz D, Gollihar J, McLellan JS, Chou CW, Javanmardi K, Finkelstein IJ, Musser JM: Molecular architecture of early dissemination and massive second wave of the SARS-CoV-2 virus in a major metropolitan area. mBio 2020, 11:6

3. Musser JM, Olsen RJ, Christensen PA, Long SW, Subedi S, Davis JJ, Gollihar J: Rapid, widespread, and preferential increase of SARSCoV-2 B.1.1.7 variant in Houston, TX, revealed by 8,857 genome sequences. medRxiv 2021, [Preprint]. doi: 10.1101/2021.03.16. 21253753

4. Olsen RJ, Christensen PA, Long SW, Subedi S, Hodjat P, Olson R, Nguyen M, Davis JJ, Yerramilli P, Saavedra MO, Pruitt L, Reppond K, Shyer MN, Cambric J, Gadd R, Thakur RM, Batajoo A, Finkelstein IJ, Gollihar J, Musser JM: Trajectory of growth of severe acute respiratory (SARS-CoV-2) syndrome coronavirus 2 variants in Houston, Texas, January through May 2021, based on 12,476 genome sequences. Am J Pathol 2021, 191:1754-1773

5. Long SW, Olsen RJ, Christensen PA, Subedi S, Olson R, Davis JJ, Saavedra MO, Yerramilli P, Pruitt L, Reppond K, Shyer MN, Cambric J, Finkelstein IJ, Gollihar J, Musser JM: Sequence analysis of 20,453 SARS-CoV-2 genomes from the Houston metropolitan area identifies the emergence and widespread distribution of multiple isolates of all major variants of concern. Am J Pathol 2021, 191: 983-992

6. Christensen PA, Olsen RJ, Long SW, Subedi S, Davis JJ, Hodjat P, Walley DR, Kinskey JC, Saavedra MO, Pruitt L, Reppond K, Shyer MN, Cambric J, Gadd R, Thakur RM, Batajoo A, Mangham R, Pena S, Trinh T, Yerramilli P, Nguyen M, Olson R, Snehal R, Gollihar J, Musser JM: Delta variants of SARS-CoV-2 cause significantly increased vaccine breakthrough COVID-19 cases in Houston, Texas. Am J Pathol 2022, 192:320-331

7. Christensen PA, Olsen RJ, Long SW, Snehal R, Davis JJ, Saavedra MO, Reppond K, Shyer MN, Cambric J, Gadd R, Thakur RM, Batajoo A, Mangham R, Pena S, Trinh T, Kinskey JC, Williams G, Olson R, Gollihar J, Musser JM: Signals of significantly increased vaccine breakthrough, decreased hospitalization rates, and less severe disease in patients with coronavirus disease 2019 caused by the Omicron variant of severe acute respiratory syndrome coronavirus 2 in Houston, Texas. Am J Pathol 2022, [Epub Ahead of Print] doi:10.1016/j.ajpath.2022.01.007

8. Viana R, Moyo S, Amoako DG, Tegally H, Scheepers C, Althaus CL, et al: Rapid epidemic expansion of the SARS-CoV-2 Omicron variant in southern Africa. medRxiv 2021, [Preprint]. doi:10.1101/2021.12. 19.21268028

9. Elliott P, Bodinier B, Eales O, Wang H, Haw D, Elliott J, Whitaker M, Jonnerby J, Tang D, Walters CE, Atchison C, Diggle PJ, Page AJ, Trotter AJ, Ashby D, Barclay W, Taylor G, Ward H, Darzi A, Cooke GS, Chadeau-Hyam M, Donnelly CA: Rapid increase in Omicron infections in England during December 2021: REACT-1 
study. Science 2022, [Epub ahead of print]. doi: 10.1126/science. abn 8347

10. Sheikh A, Kerr S, Woolhouse M, McMenamin J, Robertson C: Severity of Omicron Variant of Concern and Vaccine Effectiveness against Symptomatic Disease: National Cohort with Nested Test Negative Design Study in Scotland. Edinburgh, Scotland, University of Edinburgh, 2021

11. Meng B, Ferreira I, Abdullahi A, Kemp SA, Goonawardane N, Papa G, Fatihi S, Charles O, Collier D, Collaboration C-NBC-, Consortium TgtP J, Choi J, Hyeon Lee J, Mlcochova P, James L, Doffinger R, Thukral L, Sato K, Gupta RK: SARS-CoV-2 Omicron spike mediated immune escape, infectivity and cell-cell fusion. bioRxiv 2021, [Preprint]. doi: 10.1101/2021.12.17.473248

12. Zeng C, Evans JP, Qu P, Faraone J, Zheng Y-M, Carlin C, Bednash JS, Zhou T, Lozanski G, Mallampalli R, Saif LJ, Oltz EM, Mohler P, Xu K, Gumina RJ, Liu S-L: Neutralization and stability of SARS-CoV-2 Omicron variant. bioRxiv 2021, [Preprint]. doi: 10. 1101/2021.12.16.472934

13. Jacobsen H, Strengert M, Maass H, Ynga Durand MA, Kessel B, Harries M, Rand U, Abassi L, Kim Y, Lueddecke T, Hernandez P, Ortmann J, Heise J-K, Castell S, Gornyk D, Gloeckner S, Melhorn V, Lange B, Dulovic A, Haering J, Junker D, Schneiderhan-Marra N, Poehlmann S, Hoffmann M, Krause G, Cicin-Sain L: Diminished neutralization responses towards SARS-CoV-2 Omicron VoC after mRNA or vector-based COVID-19 vaccinations. medRxiv 2021, [Preprint]. doi: 10.1101/2021.12.21.21267898

14. Eggink D, Andeweg SP, Vennema $H$, van Maarseveen $N$, Vermaas K, Vlaemynck B, Schepers R, van Gageldonk-Lafeber AB, van den Hof S, Reusken CBEM, Knol MJ: Increased risk of infection with SARS-CoV-2 Omicron compared to Delta in vaccinated and previously infected individuals, the Netherlands, 22 November to 19 December 2021. medRxiv 2021, [Preprint]. doi: 10.1101/2021.12.20. 21268121

15. Edara V-V, Manning KE, Ellis M, Lai L, Moore KM, Foster SL, Floyd K, Davis-Gardner ME, Mantus G, Nyhoff LE, Bechnack S, Alaaeddine G, Naji A, Samaha H, Lee M, Bristow L, Hussaini L, Ciric CR, Nguyen P-V, Gagne M, Roberts-Torres J, Henry AR, Godbole S, Grakoui A, Sexton M, Piantadosi A, Waggoner JJ, Douek DC, Anderson EJ, Rouphael N, Wrammert J, Suthar MS: mRNA-1273 and BNT162b2 mRNA vaccines have reduced neutralizing activity against the SARS-CoV-2 Omicron variant. Cell Medicine Reports 2022, 3:100529

16. Zou j, Xia H, Xie X, Kurhade C, Machado RR, Weaver SC, Ren P, Shi P-Y: Neutralization against Omicron SARS-CoV-2 from previous non-Omicron infection. bioRxiv 2021, [Preprint]. doi: 2021.2012. 2020.473584

17. Ikemura N, Hoshino A, Higuchi Y, Taminishi S, Inaba T, Matoba S: SARS-CoV-2 Omicron variant escapes neutralization by vaccinated and convalescent sera and therapeutic monoclonal antibodies. medRxiv 2021, [Preprint]. doi: 10.1101/2021.12.13.21267761

18. Dejnirattisai W, Shaw RH, Supasa P, Liu C, Stuart AS, Pollard AJ, Liu X, Lambe T, Crook D, Stuart DI, Mongkolsapaya J, NguyenVan-Tam JS, Snape MD, Screaton GR: Reduced neutralisation of SARS-CoV-2 Omicron B.1.1.529 variant by post-immunisation serum. Lancet 2022, 399:234-236

19. Cameroni E, Saliba C, Bowen JE, Rosen LE, Culap K, Pinto D, et al: Broadly neutralizing antibodies overcome SARS-CoV-2 Omicron antigenic shift. Nature 2022, 602:664-670

20. Liu L, Iketani S, Guo Y, Chan JF-W, Wang M, Liu L, Luo Y, Chu H, Huang Y, Nair MS, Yu J, Chik KK-H, Yuen TT-T, Yoon C, To KK-W, Chen H, Yin MT, Sobieszczyk ME, Huang Y, Wang HH, Sheng Z, Yuen K-Y, Ho DD: Striking antibody evasion manifested by the Omicron variant of SARS-CoV-2. Nature 2022, 602:676-681

21. Planas D, Saunders N, Maes P, Benhassine FG, Planchais C, Porrot F, Staropoli I, Lemoine F, Pere H, Veyer D, Puech J, Rodary J, Bolland WH, Buchrieser J, Baele G, Dellicour S, Raymenants J,
Gorissen S, Geenen C, Vanmechelen B, Wawina T, Marti J, Cuypers L, Seve A, Hocqueloux L, Prazuck T, Loriere ES, REY F, Bruel T, Mouquet H, Andre E, Schwartz O: Considerable escape of SARS-CoV-2 Omicron to antibody neutralization. Nature 2022, 602: 671-675

22. Andrews N, Stowe J, Kirsebom F, Toffa S, Rickeard T, Gallagher E, Gower C, Kall M, Groves N, O'Connell A-M, Simons D, Blomquist PB, Zaidi A, Nash S, Aziz NIBA, Thelwall S, Dabrera G, Myers R, Amirthalingam G, Gharbia S, Barrett JC, Elson R, Ladhani SN, Ferguson N, Zambon M, Campbell CN, Brown K, Hopkins S, Chand M, Ramsay M, Bernal JL: Effectiveness of COVID-19 vaccines against the Omicron (B.1.1.529) variant of concern. medRxiv 2021, [Preprint]. doi: 10.1101/2021.12.14. 21267615

23. Yu X, Wei D, Xu W, Li Y, Li X, Zhang X, Qu J, Yang Z, Chen E: Reduced sensitivity of SARS-CoV-2 Omicron variant to boosterenhanced neutralization. Cell Discovery 2022, 8:4

24. Cele S, Jackson L, Khan K, Khoury DS, Moyo-Gwete T, Tegally H, Scheepers C, Amoako D, Karim F, Bernstein M, Lustig G, Archary D, Smith M, Ganga Y, Jule Z, Reedoy K, Cromer D, San JE, Hwa S-H, Giandhari J, Blackburn JM, Gosnell BI, Karim SSA, Hanekom W, von Gottberg A, Bhiman J, Lessells RJ, Moosa M-YS, Davenport MP, de Oliveira T, Moore PL, Sigal A; NGS-SA, Team C$\mathrm{K}$ : SARS-CoV-2 Omicron has extensive but incomplete escape of Pfizer BNT162b2 elicited neutralization and requires ACE2 for infection. medRxiv 2021, [Preprint]. doi: 10.1101/2021.12.08. 21267417

25. Cao Y, Wang J, Jian F, Xiao T, Song W, Yisimayi A, Huang W, Li Q, Wang P, An R, Wang J, Wang Y, Niu X, Yang S, Liang H, Sun H, Li T, Yu Y, Cui Q, Liu S, Yang X, Du S, Zhang Z, Hao X, Shao F, Jin R, Wang X, Xiao J, Wang Y, Xie XS: Omicron escapes the majority of existing SARS-CoV-2 neutralizing antibodies. Nature 2022, 602:657-663

26. Hansen $\mathrm{CH}$, Schelde AB, Moustsen-Helms IR, Emborg H-D, Krause TG, Moelbak K, Valentiner-Branth P; Institut TIDPGaSS: Vaccine effectiveness against SARS-CoV-2 infection with the Omicron or Delta variants following a two-dose or booster BNT162b2 or mRNA-1273 vaccination series: a Danish cohort study. medRxiv 2021, [Preprint]. doi: 10.1101/2021.12.20.21267966

27. Syed AM, Ciling A, Khalid MM, Sreekumar B, Kumar GR, Silva I, Milbes B, Kojima N, Hess V, Shacreaw M, Lopez L, Brobeck M, Turner F, Spraggon L, Taha TY, Tabata T, Chen IP, Ott M, Doudna JA: Omicron mutations enhance infectivity and reduce antibody neutralization of SARS-CoV-2 virus-like particles. medRxiv 2021, [Preprint]. doi: 10.1101/2021.12.20.21268048

28. Sheward DJ, Kim C, Ehling RA, Pankow A, Castro Dopico X, Martin DP, Reddy ST, Dillner J, Karlsson Hedestam GB, Albert J, Murrell B: Variable loss of antibody potency against SARS-CoV-2 B.1.1.529 (Omicron). bioRxiv 2021, [Preprint]. doi: 10.1101/2021. 12.19.473354

29. Haveri A, Solastie A, Ekström N, Österlund P, Nohynek H, Nieminen T, Palmu AA, Melin M: Neutralizing antibodies to SARSCoV-2 Omicron variant after 3rd mRNA vaccination in health care workers and elderly subjects and response to a single dose in previously infected adults. medRxiv 2021, [Preprint]. doi: 10.1101/2021. 12.22.21268273

30. Arien KK, Heyndrickx L, Michiels J, Vereecken K, Van Lent K, Coppens S, Pannus P, Martens GA, Van Esbroeck M, Goossens ME, Marchant A, Bartholomeeusen K, Desombere I: Three doses of the BNT162b2 vaccine confer neutralising antibody capacity against the SARS-CoV-2 B.1.1.529 (Omicron) variant of concern. medRxiv 2021, [Preprint]. doi: 10.1101/2021.12.23.21268316

31. Willett BJ, Grove J, MacLean O, Wilkie C, Logan N, De Lorenzo G, et al: The hyper-transmissible SARS-CoV-2 Omicron variant exhibits significant antigenic change, vaccine escape and a switch in cell entry mechanism. medRxiv 2022, [Preprint]. doi: 10.1101/2022.01.03. 21268111 
32. Boschi C, Colson P, Bancod A, Moal V, La Scola B: Omicron variant escapes therapeutic mAbs contrary to eight prior main VOC. bioRxiv 2022, [Preprint]. doi: 10.1101/2022.01.03.474769

33. Dejnirattisai W, Huo J, Zhou D, Zahradník J, Supasa P, Liu C, et al: Omicron-B.1.1.529 leads to widespread escape from neutralizing antibody responses. bioRxiv 2021, [Preprint]. doi: 10.1101/2021.12.03.471045

34. Banerjee A, Lew J, Kroeker A, Baid K, Aftanas P, Nirmalarajah K, Maguire F, Kozak R, McDonald R, Lang A, Gerdts V, Straus SE, Gilbert L, Li AX, Mozafarihasjin M, Walmsley S, Gingras A-C, Wrana JL, Mazzulli T, Colwill K, McGeer AJ, Mubareka S, Falzarano D: Immunogenicity of convalescent and vaccinated sera against clinical isolates of ancestral SARS-CoV-2, beta, delta, and omicron variants. bioRxiv 2022, [Preprint]. doi: 10.1101/2022.01.13. 475409

35. Ulloa AC, Buchan SA, Daneman N, Brown KA: Early estimates of SARS-CoV-2 Omicron variant severity based on a matched cohort study, Ontario, Canada. medRxiv 2021, [Preprint]. doi: 10.1101/ 2021.12.24.21268382

36. Wolter N, Jassat W, Walaza S, Welch R, Moultrie H, Groome M, Amoako DG, Everatt J, Bhiman JN, Scheepers C, Tebeila N, Chiwandire N, du Plessis M, Govender N, Ismail A, Glass A, Mlisana K, Stevens W, Treurnicht FK, Makatini Z, Hsiao N-y, Parboosing R, Wadula J, Hussey H, Davies M-A, Boulle A, von Gottberg A, Cohen C: Early assessment of the clinical severity of the SARS-CoV-2 Omicron variant in South Africa. medRxiv 2021, [Preprint]. doi: 10.1101/2021.12.21.21268116

37. SARS-CoV-2 Variants of Concern and Variants Under Investigation in England. Technical Briefing: Update on Hospitalisation and Vaccine Effectiveness for Omicron VOC-21NOV-01 (B.1.1.529). London, United Kingdom, UK Health Security Agency, 2021

38. Davies M-A, Kassanjee R, Rousseau P, Morden E, Johnson L, Solomon W, et al: Outcomes of laboratory-confirmed SARS-CoV-2 infection in the Omicron-driven fourth wave compared with previous waves in the Western Cape Province, South Africa. medRxiv 2022, [Preprint]. doi: 10.1101/2022.01.12.22269148

39. Bentley EG, Kirby A, Sharma P, Kipar A, Mega DF, Bramwell C, Penrice-Randal R, Prince T, Brown JC, Zhou J, Screaton GR, Barclay WS, Owen A, Hiscox JA, Stewart JP: SARS-CoV-2 Omicron-B.1.1.529 variant leads to less severe disease than Pango B and Delta variants strains in a mouse model of severe COVID-19. bioRxiv 2021, [Preprint]. doi: 10. $1101 / 2021.12 .26 .474085$

40. Abdelnabi R, Foo CS, Zhang X, Lemmens V, Maes P, Slechten B, Raymenants J, André E, Weynand B, Dallemier K, Neyts J: The Omicron (B.1.1.529) SARS-CoV-2 variant of concern does not readily infect Syrian hamsters. Antiviral Res 2022, 198:105253

41. Diamond M, Halfmann P, Maemura T, Iwatsuki-Horimoto K, Iida S, Kiso M, et al: The SARS-CoV-2 B.1.1.529 Omicron virus causes attenuated infection and disease in mice and hamsters. Res Sq 2021, [Preprint]. doi: 10.21203/rs.3.rs-1211792/v1

42. McMahan K, Giffin V, Tostanoski L, Chung B, Siamatu M, Suthar M, Halfmann P, Kawaoka Y, Piedra-Mora C, Martinot A, Kar S, Andersen H, Lewis MG, Barouch DH: Reduced pathogenicity of the SARS-CoV-2 Omicron variant in hamsters. bioRxiv 2022, [Preprint]. doi: 10.1101/2022.01.02.474743

43. Yuan S, Ye Z-W, Liang R, Tang K, Zhang AJ, Lu G, Ong CP, Poon VKM, Chan CC-S, Mok BWY, Qin Z, Xie Y, Sun H, Tsang JO-L, Yuen TT-T, Chik KK-H, Chan CC-Y, Cai J-P, Luo C, Lu L, Yip CC-Y, Chu H, To KK-W, Chen H, Jin D-Y, Yuen K-Y, Chan JFW: The SARSCoV-2 Omicron (B.1.1.529) variant exhibits altered pathogenicity, transmissibility, and fitness in the golden Syrian hamster model. bioRxiv 2022, [Preprint]. doi: 10.1101/2022.01.12.476031

44. SARS-CoV-2 Variants of Concern and Variants Under Investigation in England. Technical Briefing 34. London, United Kingdom, UK Health Security Agency, 2022 